\title{
Laparoscopic pancreaticoduodenectomy with excision of aberrant right hepatic artery after preoperative segmental embolization in mid-bile duct cancer
}

\author{
Yoonwon Kook ${ }^{1,4}$, Munseok Choi ${ }^{1,4}$, Jung Yup Park ${ }^{2,4}$, Ho Kyoung Hwang ${ }^{1,4}$, Woo Jung Lee ${ }^{1,4}$, Man-Deuk Kim, ${ }^{3,4}$, Chang Moo Kang \\ ${ }^{1}$ Division of HBP Surgery, Department of Surgery, Yonsei University College of Medicine, Seoul, Korea \\ 2Division of Gastroenterology, Department of Internal Medicine, Yonsei University College of Medicine, Seoul, Korea \\ ${ }^{3}$ Department of Radiology, Severance Hospital, Yonsei University College of Medicine, Seoul, Korea \\ ${ }^{4}$ Pancreatobiliary Cancer Center, Yonsei Cancer Center, Severance Hospital, Seoul, Korea
}

Laparoscopic pancreaticoduodenectomy has proven to be a safe and effective alternative to open pancreaticoduodenectomy with similar oncologic outcomes. Cases including excision of the hepatic artery with or without reconstruction during pancreaticoduodenectomy have been reported for periampullary cancer. Here we present a case of an 82-year-old patient who underwent laparoscopic pancreaticoduodenectomy following preoperative arterial embolization of an aberrant right hepatic artery arising from the superior mesenteric artery.

Keywords: Common bile duct neoplasms, Laparoscopy, Pancreaticoduodenectomy, Hepatic artery, Embolization
Received May 21, 2020

Revised November 23, 2020

Accepted January 14, 2021

Corresponding author

Chang Moo Kang

Department of Surgery, Yonsei

University College of Medicine,

Ludlow Faculty Research Building

\#201, 50 Yonsei-ro, Seodaemun-gu,

Seoul 03722, Korea

Tel: $+82-2-2228-2135$

Fax: +82-2-313-8289

E-mail: cmkang@yuhs.ac

ORCID:

https://orcid.org/0000-0002-5382-4658

Copyright () The Korean Society of Endoscopic and Laparoscopic Surgeons.
This is an Open Access article distributed under the terms of the Creative Commons Attribution Non-Commercial License (http:/l creativecommons.org/licenses/by-nc/4.0/) which permits unrestricted non-commercial use, distribution, and reproduction in any medium, provided the original work is properly cited.

\section{INTRODUCTION}

Although still debated, the literature and current practices show that laparoscopic pancreaticoduodenectomy (LPD) is feasible, safe, and even oncologically effective in managing periampullary cancer. A recent meta-analysis comparing open pancreaticoduodenectomy (PD) and LPD demonstrated that LPD is as safe and effective as open PD, with similar perioperative and longterm oncologic outcomes [1], and with shorter hospital stay, less intraoperative bleeding, and fewer postoperative complications. In addition, a randomized control study also demonstrated that LPD was associated with a shorter length of hospital stay and a more favorable postoperative course with similar oncologic outcomes [2].

It has also been suggested that LPD with venous vascular resection is feasible and safe with comparable perioperative results [3]. However, there is a demand for advanced surgical techniques and experience to prevent unexpected intraoperative accidents. Therefore, standardization remains difficult, and this plays a role in limiting the indication for LPD. There are currently no reports of LPD with combined hepatic artery resection and reconstruction, suggesting that this clinical situation is one of the technical limitations in expanding the indication for LPD.

Hepatic perfusion is crucial during LPD, and some studies 
even suggest that there is potential collateral hepatic flow after division of the proper hepatic artery [4]. Hepatic collateral flow depends on the individual vascular anatomical characteristics and the duration since the injury. However, in some critical cases, collateral flow develops through the liver suspensory ligaments and small retroperitoneal vessels, which take time to develop sufficient hepatic perfusion to avoid hepatic necrosis, especially after PD. This intraoperative insult may worsen postoperative recovery after $\mathrm{PD}$.

In this report, we present the case of an 82-year-old female patient who successfully underwent LPD with excision of the right hepatic artery following preoperative short segment embolization for mid-bile duct cancer.

\section{CASE REPORT}

\section{Case presentation}

An 82-year-old woman was admitted to our hospital with general weakness, abdominal pain, and fever in March 2020. The patient's general appearance was good and her vital signs were within normal limits, with the exception of a fever of $39.3^{\circ} \mathrm{C}$. The patient had a history of hypertension, old cerebral infarction, and nontuberculous mycobacterial pneumonia.

Laboratory findings showed a complete blood count with a shift to left (neutrophil, 92\%; reference, 39\%-74\%), elevated liver profile including aspartate transaminase $(2,616 \mathrm{IU} / \mathrm{L}$; reference, 13-34 IU/L), alanine transaminase (499 IU/L; reference, 5-46 IU/ L), elevated total bilirubin ( $3.2 \mathrm{mg} / \mathrm{dL}$; reference, $0.4-1.5 \mathrm{mg} / \mathrm{dL})$, direct bilirubin (1.8 mg/dL; reference, $0.1-0.4 \mathrm{mg} / \mathrm{dL})$, alkaline phosphatase (396 IU/L; reference, 50-155 IU/L), and gamma-glutamyl transpeptidase (347 IU/L; reference, 7-35 IU/L). The initial serum carbohydrate antigen 19-9 (CA 19-9) level was 143 U/mL (reference, 0.0-34 U/mL), which increased to 2,308 U/mL during the preoperative evaluation period.

Abdominal magnetic resonance imaging showed diffuse bile duct dilatation with a distended gallbladder resulting from an occlusive mass effect in the suprapancreatic common bile duct (CBD) area. In addition, the tumor seemed to be abutting the portal vein, and there was an aberrant right hepatic artery (aRHA) rising from the superior mesenteric artery (SMA) in the computed tomography (CT) scan (Fig. 1A). Positron emission tomography-CT scans showed not only intense fluorodeoxyglucose uptake in the hepatoduodenal ligament area but also intense
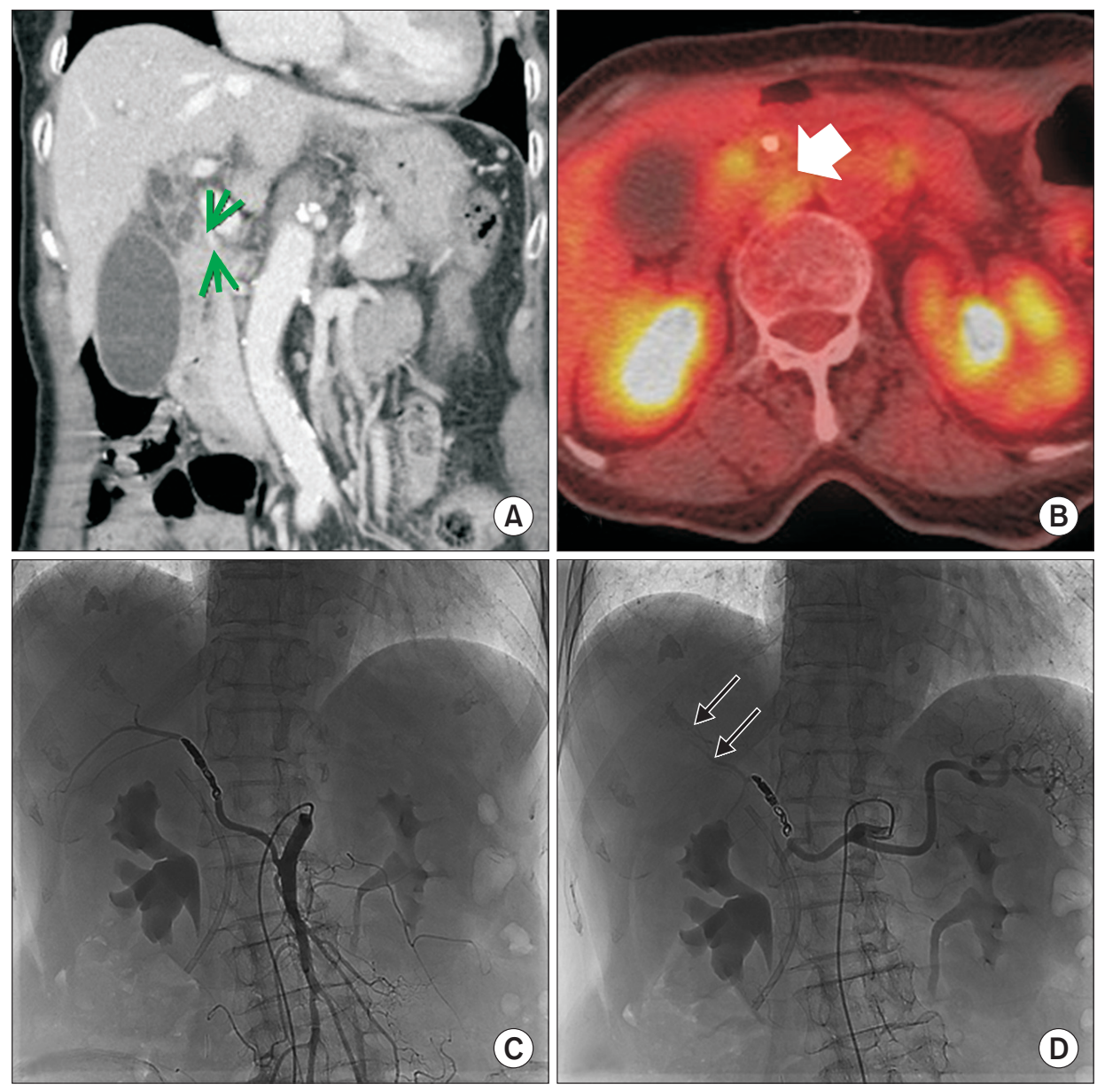

Fig. 1. Preoperative evaluation. (A) Common bile duct cancer abutting to the portal vein and right aberrant hepatic artery (green arrows). (B) Paraaortic lymph node with considerable fluorodeoxyglucose uptake was noted to be suspicious metastatic lymph node (thick white arrow). (C) Preoperative angiography and segmental embolization of aberrant right hepatic artery (aRHA) were performed. (D) Subsequent celiac angiogram demonstrated the well perfusion of the right liver (black arrows) through the left hepatic artery even after occlusion of aRHA. 
signals in the paraaortic areas (Fig. 1B). No other evidence of systemic metastasis was observed. Subsequent endoscopic retrograde cholangiopancreatography with biopsy and endoscopic retrograde biliary drainage catheter insertion was performed, and the biopsy indicated a well-differentiated adenocarcinoma. Her general condition and preoperative evaluation allowed her to be considered as a candidate for PD with combined excision of aRHA and paraaortic lymph node clearance.

\section{Preoperative celiac and superior mesentery artery angiography with embolization of the aberrant right hepatic artery}

Angiography was performed on March 3, 2020, to confirm the aRHA from the SMA and the left hepatic artery, with collateral flow to the right liver from the celiac artery (Fig. 1C). Subsequent embolization of the aRHA was performed to further increase the potential collateral blood flow in case of combined excision of the involved aRHA during the surgical procedure (Fig. 1D). A follow-up CT scan 10 days later revealed that there was no ischemic change to the right liver after embolization of the aRHA, which was evidence of sufficient collateral blood flow.

\section{Operation}

On March 27, 2020, 2 weeks after the embolization, the patient underwent laparoscopic PD with tangential resection of the portal vein and combined excision of the aRHA. Some enlarged lymph nodes stationed around the celiac trunk and in the retroperitoneum were sent for frozen sectioning, all of which were found to be free from carcinoma. Intraoperative paraaortic lymph node sampling was performed, and the frozen sections were reported to be free from carcinoma (Fig. 2A). Following confirmation that there were no metastatic distant lymph nodes, the surgical procedure continued, and the origin of the aRHA was ligated using vascular Endo GIA (Medtronic, Minneapolis, MN, USA) (Fig. 2B). The upper resection margin of the bile duct was sent for frozen sectioning, and no cancer involvement was reported. A small part of the portal vein was adhered to the tumor and could not be separated. Tangential vascular partial resection was performed, and primary repair was performed using a 5-0 monofilament non-absorbable suture after portal
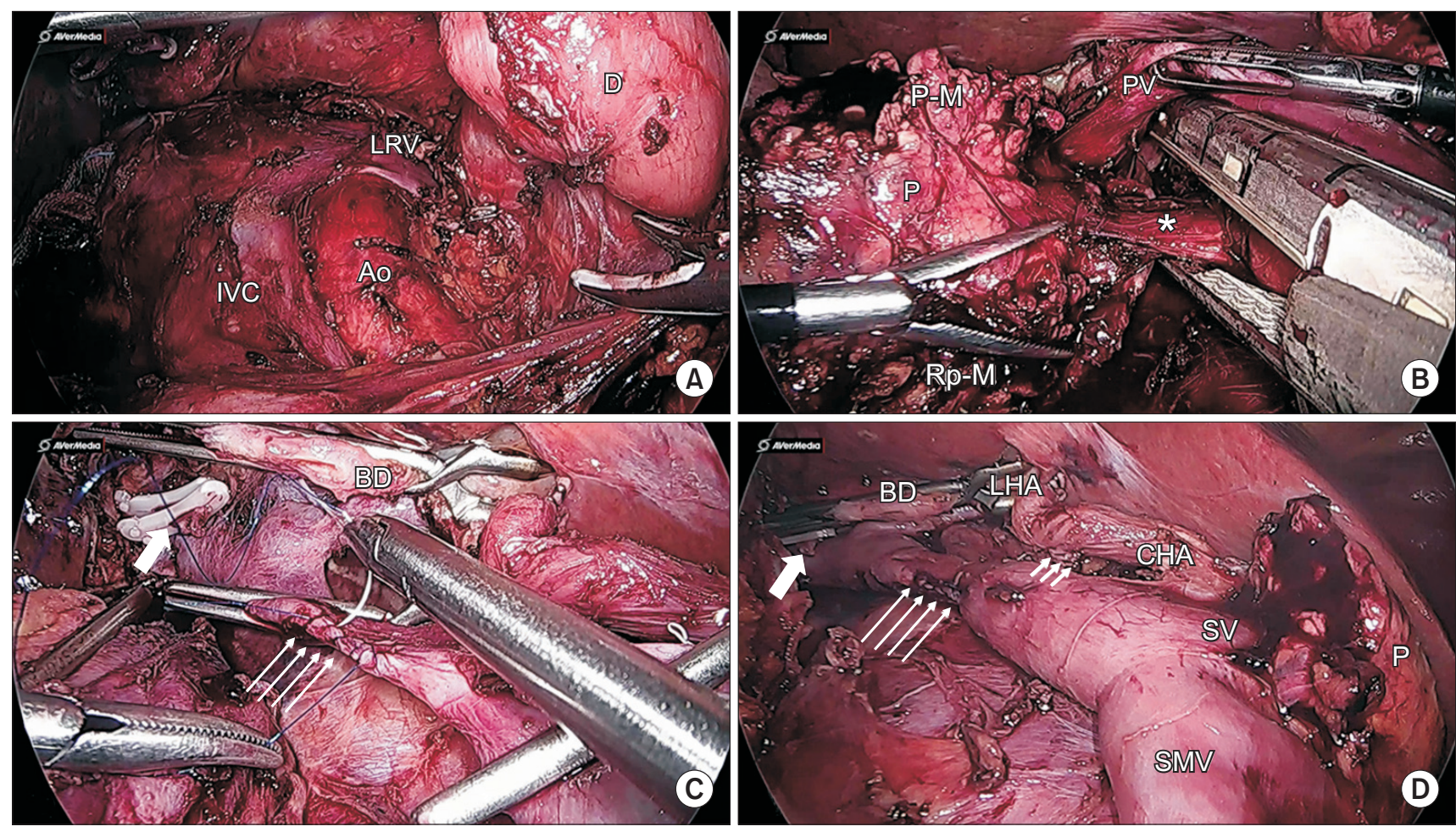

Fig. 2. Operative view. (A) Paraaortic lymph node sampling and frozen-section biopsy. (B) Division of the origin of aberrant right hepatic artery (asterisk) by Endo GIA (Medtronic, Minneapolis, MN, USA). (C) Tangential resection of the portal vein (PV) after bulldog vascular clamp (long thin white arrows). (D) Final surgical field resection phase of pancreaticoduodenectomy. Note the divided distal portion of aberrant right hepatic artery (thick white arrows), and primary repair site of the resected PV (long thin white arrows). D, duodenum; LRV, left renal vein; IVC, inferior renal vein; A0, aorta; P-M, pancreatic cut surface; P, remnant pancreas; Rp-M, retroperitoneal or superior mesenteric artery lateral margin area; BD, resected bile duct; LHA, left hepatic artery; CHA, common hepatic artery; SV, splenic vein; SMV, superior mesenteric vein. 
vein cross-clamping with two bull-dog clamps (Fig. 2C). Upon completion of the resection phase (Fig. 2D), subsequent pancreatojejunostomy and hepaticojejunostomy were performed in the usual manner. The surgery took 450 minutes and there was 250 $\mathrm{mL}$ of blood loss.

\section{Postoperative course and pathological examination}

The patient recovered without significant surgery-related complications. Clinically, nonrelevant postoperative pancreatic fistula (biochemical leak) was noted, and all of the surgical drains were removed on postoperative day 5. The serum CA 19-9 level was $165 \mathrm{U} / \mathrm{mL}$ on postoperative day 7. Postoperative conservative management including nutritional support was necessary to improve the patient's general condition, and she was finally discharged on postoperative day 24 . The patient visited the outpatient department for an 8-month follow-up after surgery on November 23, 2020. She was in good general condition with laboratory results within normal range and her CT scan showed no evidence of recurrence.

Final pathological examination revealed that the tumor was a 2.3-cm sized adenocarcinoma (moderately differentiated) of the CBD. The tumor invaded the bile duct wall and periductal fat tissue at a depth of $7 \mathrm{~mm}$ (pathologic T2), but no extension to the pancreas was found. Lymphovascular invasion was absent, but perineural invasion was noted. Fortunately, the resected portal vein and right hepatic artery were found to be free from cancer. It was noted that none of the resection margins, including the CBD, pancreatic duct, retroperitoneal SMA lateral margin, superior mesenteric vein-portal vein groove, anterior surface, posterior surface, and the duodenum, were invaded by malignant cells. No metastatic lymph nodes were identified.

\section{DISCUSSION}

Margin-negative resection is very important in the treatment of bile duct cancer. In this case, PD was considered curative treatment for the patient. Considering the patient's age, segmental resection of the bile duct could have been an alternative to PD. However, the intraoperative distal margin of the resected bile duct based on frozen-section biopsy during the operation might have resulted in subsequent PD and prolongation of the operation, which could have negatively impacted the patient's recovery. In addition, a recent Korea-Japan international collaboration study [5] showed impaired long-term oncologic outcomes of segmental resection of the bile duct when compared with PD for the treatment of mid-bile duct cancer. Although the current report is not a randomized control study, it provides important insight into surgical decision-making for mid-bile duct cancer.

Although laparoscopic PD is controversial, the accumulation of experience demonstrates that LPD is safe and effective, showing improved perioperative surgical outcomes with comparable long-term oncologic outcomes. Therefore, well-selected patients can gain clinical benefit from LPD in clinical oncology. In addition, recent literature also suggests that LPD is appropriate for the treatment of the elderly [6]. Generally, age itself is not a contraindication for PD, and functional performance status is considered more important in patient selection. Therefore, careful patient selection is required when offering surgery to this age group. From the viewpoint of the current aged society, this issue will be discussed further in the near future.

The oncologic impact of combined hepatic arterial resection in the treatment of bile duct cancer remains controversial. However, several studies have shown favorable long-term oncologic outcomes, suggesting the oncologic role of aggressive surgery in treating advanced bile duct cancer [7]. Although aRHA can be safely resected during PD, there were several reasons for choosing preoperative embolization of aRHA for the current patient. First, laparoscopic dissection of the aRHA from the abutting tumor would result in a long operation time, which could negatively impact the recovery of this patient. Second, there was a potential risk of postoperative hemorrhage from the dissected aRHA as a result of arterial denuding during the surgical procedure. Third, laparoscopic arterial reconstruction (end-to-end) is considered technically difficult and unstable. Therefore, combined excision of the aRHA was initially planned for procedural safety, simplicity, and curability. Lastly, preoperative angiogram demonstrated sufficient collateral vessels to the right liver, and as such, combined excision of the aRHA could have been feasible without preoperative arterial embolization. However, ischemic hepatic damage during the operation could have resulted in dismal recovery after surgery in this octogenarian patient. A potential reservoir of collateral blood flow to the right liver was expected to be further developed after embolization of aRHA. Therefore, taking all of this into consideration, preoperative embolization of the aRHA followed by LPD was decided to enhance the procedural safety for this patient.

Aside from the current report, we also consider the situation in which the distal bile duct cancer is invading the common hepatic artery or the proper hepatic artery? Is this a contraindication for surgical resection? Although neoadjuvant chemotherapy is widely accepted in borderline and locally advanced pancreatic cancer [8], there is still a lack of evidence to support neoadjuvant chemotherapy followed by aggressive surgical extirpation in advanced bile duct cancer [9]. We consider that this preoperative arterial embolization may function to enhance the hepatic collateral flow may work such that an aggressive surgical approach with combined hepatic arterial resection can be performed selectively [4]. Therefore, the present case demonstrates the safety and technical feasibility of LPD with hepatic arterial excision after preopera- 
tive hepatic artery embolization in well-selected advanced bile duct cancer. Cloyd et al. [10] first reported preoperative embolization of a tumor encasing the right hepatic artery in patients with $\mathrm{PD}$; however, to the best of our knowledge, the present case is the first published report in the field of LPD.

In summary, in specific cases where the hepatic artery is invaded by periampullary cancer, LPD with excision of the involved hepatic artery without reconstruction is both safe and feasible. However, patient selection is key in order to reduce perioperative risk and ensure safety after hepatic artery excision. Therefore, preoperative short segment embolization may be a potential approach to allow safe LPD with hepatic artery excision. Further experiences should follow.

\section{NOTES}

\section{Ethical statements}

This study was approved by the Institutional Review Board of Yonsei University Health System with a waiver of informed consent (No. 4-2020-1110).

\section{Authors' contributions}

Conceptualization: $\mathrm{CMK}$

Data curation: YK, MC

Formal analysis: $\mathrm{YK}, \mathrm{MC}, \mathrm{CMK}$

Funding acquisition: $\mathrm{CMK}$

Investigation: $\mathrm{YK}, \mathrm{MC}$

Methodology: JYP, HKH, MDK, WJL

Project administration: $\mathrm{CMK}, \mathrm{MDK}$

Visualization: $\mathrm{YK}, \mathrm{MC}, \mathrm{CMK}$

Writing-original draft: $\mathrm{YK}, \mathrm{MC}, \mathrm{CMK}$

Writing-review \& editing: YK, MC, CMK

All authors read and approved the final manuscript.

\section{Conflict of interest}

All authors have no conflicts of interest to declare.

\section{ORCID}

Yoonwon Kook, https://orcid.org/0000-0002-0756-738X
Munseok Choi, https://orcid.org/0000-0002-9844-4747

Jung Yup Park, https://orcid.org/0000-0003-0110-8606

Ho Kyoung Hwang, https://orcid.org/0000-0003-4064-7776

Woo Jung Lee, https://orcid.org/0000-0001-9273-261X

Man-Deuk Kim, https://orcid.org/0000-0002-3575-5847

Chang Moo Kang, https://orcid.org/0000-0002-5382-4658

\section{REFERENCES}

1. Pang L, Kong J, Wang Y, Zhang Y. Laparoscopic versus open pylorus-preserving pancreatoduodenectomy: the first meta-analyse of retrospective matched cases. Acta Cir Bras 2018;33:40-48.

2. Poves I, Burdío F, Morató O, et al. Comparison of perioperative outcomes between laparoscopic and open approach for pancreatoduodenectomy: the PADULAP randomized controlled trial. Ann Surg 2018;268:731-739.

3. Yu XZ, Li J, Fu DL, et al. Benefit from synchronous portal-superior mesenteric vein resection during pancreaticoduodenectomy for cancer: a meta-analysis. Eur J Surg Oncol 2014;40:371-378.

4. Yasuda Y, Larsen PN, Ishibashi T, Yamashita K, Toei H. Resection of hilar cholangiocarcinoma with left hepatectomy after pre-operative embolization of the proper hepatic artery. HPB (Oxford) 2010;12:147152.

5. Hayashi H, Jang JY, Kim KS, et al. Comparison of pancreaticoduodenectomy and bile duct resection for middle bile duct cancer: a multi-center collaborating study of Japan and Korea. J Hepatobiliary Pancreat Sci 2020;27:289-298.

6. Meng L, Xia Q, Cai Y, et al. Impact of patient age on morbidity and survival following laparoscopic pancreaticoduodenectomy. Surg Laparosc Endosc Percutan Tech 2019;29:378-382.

7. Banales JM, Cardinale V, Carpino G, et al. Expert consensus document: cholangiocarcinoma: current knowledge and future perspectives consensus statement from the European Network for the Study of Cholangiocarcinoma (ENS-CCA). Nat Rev Gastroenterol Hepatol 2016;13:261-280.

8. Oba A, Ho F, Bao QR, Al-Musawi MH, Schulick RD, Del Chiaro M. Neoadjuvant treatment in pancreatic cancer. Front Oncol 2020;10: 245.

9. Imperatori M, D’Onofrio L, Marrucci E, Pantano F, Zoccoli A, Tonini G. Neoadjuvant treatment of biliary tract cancer: state-of-the-art and new perspectives. Hepat Oncol 2016;3:93-99.

10. Cloyd JM, Chandra V, Louie JD, Rao S, Visser BC. Preoperative embolization of replaced right hepatic artery prior to pancreaticoduodenectomy. J Surg Oncol 2012;106:509-512. 